\title{
Transient heat transfer in a rarefied binary gas mixture confined between parallel plates due to a sudden small change of wall temperatures
}

\author{
Polikarpov A. Ph. ${ }^{\mathrm{a}, *}$, Ho Minh Tuan ${ }^{\mathrm{b}}$, Graur I. ${ }^{\mathrm{c}}$ \\ ${ }^{a}$ Physics Department, Ural Federal University, \\ Lenina str. 51, 620000, Ekaterinburg, Russia \\ ${ }^{b}$ James Weir Fluids Laboratory, Department of Mechanical and Aerospace Engineering, \\ University of Strathclyde, Glasgow G1 1 XJ, UK \\ ${ }^{c}$ Aix Marseille Université - Polytech Marseille, \\ Département de Mécanique Energétique - IUSTI, UMR CNRS 7343, \\ 5 rue Enrico Fermi, 13453 Marseille cedex 13, France
}

\begin{abstract}
Transient behavior of the heat transfer through binary gas mixture, confined between two infinite parallel plates, caused by the sudden change of the plates' temperatures, is studied for two monoatomic gas mixtures: Ne-Ar and He-Ar. The walls' temperature changes are considered small compared to the equilibrium temperature of the system, so the McCormack kinetic model is used for the numerical simulations. The time evolution of the main macroscopic parameter is investigated for various species concentrations and for different gas rarefactions ranging from near the free molecular to slip flow regime. It is found that the mixture heat flux takes several characteristic times, which is defined by the distance between the plates over the most probable molecular speed, to achieve its new equilibrium state. This time of the steady state flow establishment depends strongly on the gas rarefaction, mixture nature and composition.
\end{abstract}

Keywords: rarefied gas, Boltzmann equation, binary mixture

\section{Introduction}

The investigation of the time response of a rarefied gas confined between two parallel plates to a sudden change in the plates' temperature is important from the both fundamental and practical points of view. A gas between two surfaces is considered rarefied when the molecular mean free path is comparable to (or larger than) the gap between the surfaces. For accurate modeling of the rarefied

\footnotetext{
*Principal Corresponding Author

** Corresponding Author

Email addresses: alexey.polikarpov@gmail.com (Polikarpov A. Ph.), irina.martin@univ-amu.fr (Graur I.)
} 
gas behaviors the approaches, which take into account the molecular structure of the gas must be applied.

In the case of the constant surface temperatures the heat transfer through a single gas, confined between two parallel plates, was exhaustively investigated by many authors using both continuum and kinetic approaches, see Ref. [1, 2, 3, 4, $5,6,7]$. Several works were devoted to the analysis of the steady state heat flux through the binary gas mixture in the two plates geometry, see Refs. [8, 9, 10], where the analytical expressions for the heat transfer in the free molecular and slip flow regimes were suggested [10]. However in the most practical problems the surface temperatures can vary and so the problem becomes time dependent. The transient properties of the heat flux were studied in Refs. [11, 12, 13, $14,15,16,17,18]$ in the case of a single gas. The collisionless regime was simulated in Refs. $[12,14]$, and the semi-analytical expressions were provided for the macroscopic gas parameters in the case of the sudden surfaces' heating (or cooling) and the good agreement between proposed expressions and the DSMC simulations [14] was found. In Refs. $[15,16,19]$ the oscillatory changes in the surface temperatures were considered. However only one publication, as far as the authors are aware, is devoted to the simulation of the transient flow behavior of the gas mixture confined between two coaxial cylinders [20].

In this work we consider the effects of a step change in walls' temperatures on the gas mixture confined between the two parallel plates. We determine how long and in what manner a new steady state condition is achieved. We provide also the time needed for a mixture to go to its new equilibrium state. The influences of gas rarefaction and molar concentration on this process are analyzed for two mixtures, with the similar (Ne-Ar) and disparate (He-Ar) molecular masses.

\section{Problem statement}

Let us consider a binary mixture of monatomic gases, where the mass of a molecule of the first (second) species is $m_{1}\left(m_{2}\right)$, and the corresponding number density is $n_{1}\left(n_{2}\right)$. Without loss of generality, we assume $m_{1}<m_{2}$. The gaseous mixture is confined between two infinitely long parallel plates situated at $y^{\prime}= \pm H / 2$, see Fig. 1 . Both plates are stationary and are kept initially at the same temperature of $T_{0}$. Then, instantaneously the temperature of the both plates changes: the temperature of the down plate decreases to $T_{0}-\Delta T / 2$ and the temperature of the upper plate increases up to $T_{0}+\Delta T / 2$. We assume that the temperature difference $\Delta T$ is much smaller than the equilibrium gas temperature $T_{0}\left(\Delta T \ll T_{0}\right)$, so the gaseous mixture deviates only slightly from its thermodynamic equilibrium and the McCormack model [21] can be applied to simulate the relaxation of the mixture to a new equilibrium state.

The most probable molecular velocity of the mixture is:

$$
v_{0}=\sqrt{\frac{2 k T_{0}}{m}},
$$


where $k$ is the Boltzmann constant and $m=C_{0} m_{1}+\left(1-C_{0}\right) m_{2}$ is the mean molecular mass of the mixture. Here, $C_{0}$ is initial equilibrium molar concentration of the lighter species,

$$
C_{0}=\frac{n_{01}}{n_{01}+n_{02}},
$$

and $n_{0 \alpha}$ is the equilibrium number density of species $\alpha(\alpha=1,2)$. The equilibrium number density of the mixture is $n_{0}=n_{01}+n_{02}$. The most probable molecular velocity of species $\alpha$ is defined as

$$
v_{0 \alpha}=\sqrt{\frac{2 k T_{0}}{m_{\alpha}}}
$$

We can define the mixture macroscopic velocity $u_{y}^{\prime}$ as

$$
u_{y}^{\prime}=\frac{m_{1} n_{01} u_{1 y}^{\prime}+m_{2} n_{02} u_{2 y}^{\prime}}{m_{1} n_{01}+m_{2} n_{02}}
$$

Here we are interested in the dimensionless heat flux and mixture velocity

$$
q_{y}=\frac{q_{y}^{\prime}}{p_{0} v_{0}} \frac{T_{0}}{\Delta T}, \quad u_{y}=\frac{u_{y}^{\prime}}{v_{0}} \frac{T_{0}}{\Delta T}
$$

as well as in the profiles of the deviated temperature, density, and concentration

$$
T=\frac{T^{\prime}-T_{0}}{\Delta T}, \quad n=\frac{n^{\prime}-n_{0}}{n_{0}} \frac{T_{0}}{\Delta T}, \quad C=\frac{C^{\prime}-C_{0}}{C_{0}} \frac{T_{0}}{\Delta T} .
$$

It is to note that the macroscopic parameters, defined by Eqs. (5), (6) are time $(t)$ and space $(y)$ dependent.

Here the molar concentration of the mixture is defined as

$$
C^{\prime}=\frac{n_{1}^{\prime}}{n_{1}^{\prime}+n_{2}^{\prime}}
$$

and $n_{\alpha}^{\prime}(\alpha=1,2)$ is the deviated number density of species $\alpha, p_{0}$ is the equilibrium pressure of the mixture, $p_{0}=n_{0} k T_{0}$. The gas rarefaction is characterized by the rarefaction parameter which is defined as

$$
\delta=\frac{H}{\ell}, \quad \ell=\frac{\mu v_{0}}{p_{0}},
$$

where $\ell$ is the equivalent mean free path, $\mu=\mu_{1}+\mu_{2}$ is the viscosity of the mixture at the equilibrium temperature $T_{0}$ and its expression is given in Appendix A. 


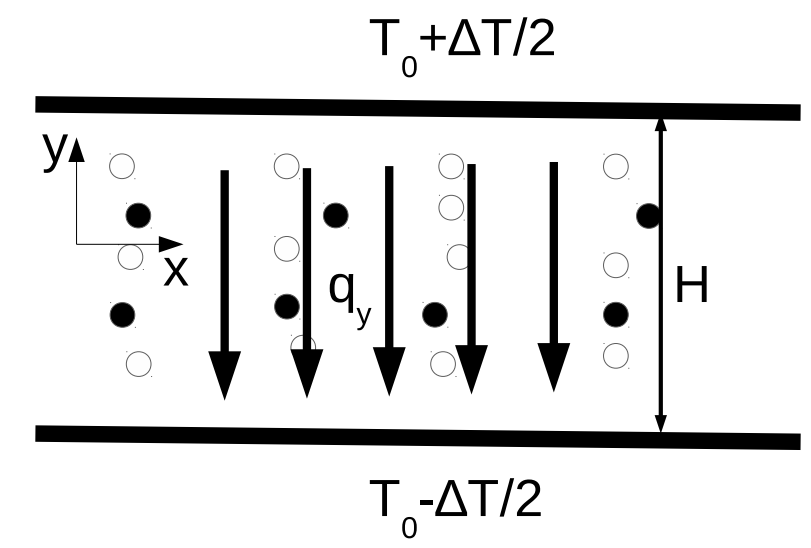

Figure 1: Flow geometry

\section{Kinetic equation}

The Boltzmann kinetic equation is used to simulate the transient heat transfer through the gas mixture at arbitrary gas rarefaction. Since only small temperature difference between the plates' surfaces is considered, the Boltzmann equation can be linearized by classical manner as

$$
f_{\alpha}=f_{\alpha}^{M}(\mathbf{v})\left[1+h_{\alpha} \Delta T / T_{0}\right], \quad \alpha=1,2,
$$

where $f_{\alpha}^{M}$ is Maxwellian equilibrium distribution function:

$$
f_{\alpha}^{M}(\mathbf{v})=n_{0 \alpha}\left(\frac{m_{\alpha}}{2 \pi k T_{0}}\right)^{3 / 2} \exp \left(-\frac{m_{\alpha} \mathbf{v}^{2}}{k T_{0}}\right)
$$

where $\mathbf{v}$ is the molecular velocity $\alpha$ and $h_{\alpha}$ is the perturbation function of species $\alpha$, which obeys the following two coupled linearized Boltzmann equations [22]

$$
\frac{\partial h_{\alpha}}{\partial t^{\prime}}+v_{y} \frac{\partial h_{\alpha}}{\partial y^{\prime}}=\sum_{\beta=1}^{2} \hat{Q}_{\alpha \beta} h, \quad \alpha=1,2,
$$

where $\hat{Q}_{\alpha \beta} h$ is the linearized collision term. Here the McCormack model [21] is used for the simulation of the collision term.

It is convenient to introduce, in addition to the already defined by Eqs. (5) and (6) dimensionless variables, the following dimensionless quantities

$$
t=\frac{t^{\prime}}{t_{0}}, \quad y=\frac{y^{\prime}}{H}, \quad \mathbf{c}_{\alpha}=\frac{\mathbf{v}}{v_{0 \alpha}}, \quad P_{\alpha y y}=-\frac{T_{0}}{2 \Delta T p_{0 \alpha}} P_{\alpha y y}^{\prime},
$$

where $\mathbf{c}_{\alpha}$ is molecular velocity of the species $\alpha, P_{\alpha y y}$ is its partial perturbed pressure, $p_{0 \alpha}=n_{0 \alpha} k T_{0}$ is its partial initial pressure. The time in Eqs. (12) is 
scaled by the characteristic time, which is defined as a mean time needed for a molecule to cross the gap between two plates

$$
t_{0}=H / v_{0} .
$$

It is worth to note that this characteristic time is close to the acoustic time, which can be defined here as $t_{a}=H / \sqrt{\gamma k T_{0} / m}$ ( $\gamma$ is the heat capacity ratio). The ratio between these two times in the case of the monoatomic gases is $t_{0} / t_{a}=$ $\sqrt{2 / \gamma}=1.095$.

For the results analysis, see discussion in Section 5.3, the mean time between the collision is introduced as

$$
t_{c}=\frac{\ell}{v_{0}} .
$$

This time can be related to the time-of-flight of molecules in the gap between the plates (or characteristic time) $t_{0}$ as

$$
t_{c}=\frac{t_{0}}{\delta} .
$$

With the introduced above dimensionless variables the linearized Boltzmann equation (11) for species $\alpha$ becomes

$$
\frac{\partial h_{\alpha}}{\partial t} \sqrt{\frac{m_{\alpha}}{m}}+c_{\alpha y} \frac{\partial h_{\alpha}}{\partial y}=\sqrt{\frac{m_{\alpha}}{m}} \delta A \sum_{\beta=1}^{2} \hat{L}_{\alpha \beta} h, \quad A=\left(\frac{C_{0}}{\gamma_{1}}+\frac{1-C_{0}}{\gamma_{2}}\right) .
$$

The expression for the McCormack collisional terms as well as the expressions for the parameters $\gamma_{1}$ and $\gamma_{2}$, the collision frequencies, are provided in Appendix A.

When the perturbation functions $h_{\alpha}(\alpha=1,2)$ are known from the solution of Eq. (16), the macroscopic flow characteristics are calculated as follows

$$
\begin{aligned}
n_{\alpha} & =\frac{1}{\pi^{3 / 2}} \sqrt{\frac{m}{m_{\alpha}}} \int h_{\alpha} \exp \left(-\boldsymbol{c}_{\alpha}^{2}\right) d \boldsymbol{c}_{\alpha}, \\
u_{\alpha y} & =\frac{1}{\pi^{3 / 2}} \sqrt{\frac{m}{m_{\alpha}}} \int h_{\alpha} c_{\alpha y} \exp \left(-\boldsymbol{c}_{\alpha}^{2}\right) d \boldsymbol{c}_{\alpha}, \\
P_{\alpha y y} & =\frac{1}{\pi^{3 / 2}} \int h_{\alpha}\left(c_{y \alpha}^{2}-\frac{1}{3} c_{\alpha}^{2}\right) \exp \left(-\boldsymbol{c}_{\alpha}^{2}\right) d \boldsymbol{c}_{\alpha}, \\
T_{\alpha} & =\frac{1}{\pi^{3 / 2}} \int h_{\alpha}\left(\frac{2}{3} c_{\alpha}^{2}-1\right) \exp \left(-\boldsymbol{c}_{\alpha}^{2}\right) d \boldsymbol{c}_{\alpha}, \\
q_{\alpha y} & =\frac{1}{\pi^{3 / 2}} \sqrt{\frac{m}{m_{\alpha}}} \int h_{\alpha} c_{\alpha y}\left(\boldsymbol{c}_{\alpha}^{2}-\frac{5}{2}\right) \exp \left(-\boldsymbol{c}_{\alpha}^{2}\right) d \boldsymbol{c}_{\alpha} .
\end{aligned}
$$

The dimensionless macroscopic quantities of the binary gaseous mixture are 
defined as follows

$$
\begin{aligned}
u_{y} & =\left(C_{0} m_{1} u_{1 y}+\left(1-C_{0}\right) m_{2} u_{2 y}\right) / m, \\
T & =C_{0} T_{1}+\left(1-C_{0}\right) T_{2}, \\
q_{y} & =C_{0} q_{1 y}+\left(1-C_{0}\right) q_{2 y}, \\
n & =C_{0} n_{1}+\left(1-C_{0}\right) n_{2}, \\
C & =\left(1-C_{0}\right)\left(n_{1}-n_{2}\right) .
\end{aligned}
$$

As it was underlined previously all quantities in Eq. (18) depend on time and space variables.

The Maxwell diffuse-specular boundary condition is used to describe the gas-wall interaction:

$$
\begin{aligned}
h_{\alpha(y=1 / 2)}^{+} & =\left(1-a_{\alpha}^{y=1 / 2}\right) h_{\alpha(y=1 / 2)}^{-}+a_{\alpha}^{y=1 / 2}\left(n_{\alpha}^{y=1 / 2}-\frac{3}{4}+\frac{1}{2} c_{\alpha}^{2}\right), \\
h_{\alpha(y=-1 / 2)}^{+} & =\left(1-a_{\alpha}^{y=-1 / 2}\right) h_{\alpha(y=-1 / 2)}^{-}+a_{\alpha}^{y=-1 / 2}\left(n_{\alpha}^{y=-1 / 2}+\frac{3}{4}-\frac{1}{2} c_{\alpha}^{2}\right),
\end{aligned}
$$

where $\alpha=1,2, a_{\alpha}^{y= \pm 1 / 2}$ are the accommodation coefficients of specie $\alpha$ on the upper and down plates, respectively, the superscripts + and - of the perturbation function $h_{\alpha}$ in Eq. (19) refer to the outgoing and incoming molecules with respect to the plates' surfaces, respectively, $\alpha=1,2, n_{\alpha}^{y= \pm 1 / 2}$ is the number density on the plates' surfaces, which is calculated from non-penetration conditions:

$$
\begin{aligned}
n_{\alpha}^{y=1 / 2} & =-\frac{1}{4}+\frac{2}{\pi} \int_{c_{\alpha y>0}} h_{\alpha(y=1 / 2)}^{-} \exp \left(-c_{\alpha}^{2}\right) c_{\alpha y} d c_{\alpha}, \\
n_{\alpha}^{y=-1 / 2} & =+\frac{1}{4}-\frac{2}{\pi} \int_{c_{\alpha y<0}} h_{\alpha(y=-1 / 2)}^{-} \exp \left(-c_{\alpha}^{2}\right) c_{\alpha y} d c_{\alpha} .
\end{aligned}
$$

At the initial time $t=0$, the specie perturbation function $h_{\alpha}$ is zero everywhere, so all the deviated macroscopic quantities Eqs. (5), (6) are set equal to 0 .

\section{Method of solution}

The discrete velocity method (DVM) [23] is used to solve the McCormack kinetic equation (16) for each species $\alpha=1,2$. To reduce computational effort, the $c_{\alpha z}$ variable is eliminated by introducing the reduced functions of $h_{\alpha}$ [24] $[25,26,27]$ :

$$
\begin{aligned}
& \Phi_{\alpha}=\frac{1}{\sqrt{\pi}} \sqrt{\frac{m}{m_{\alpha}}} \int h_{\alpha} \exp \left(-c_{\alpha z}^{2}\right) d c_{\alpha z}, \\
& \Psi_{\alpha}=\frac{1}{\sqrt{\pi}} \sqrt{\frac{m}{m_{\alpha}}} \int h_{\alpha} c_{\alpha z}^{2} \exp \left(-c_{\alpha z}^{2}\right) d c_{\alpha z} .
\end{aligned}
$$


For the considered one-dimensional heat transfer problem the number of velocities in the molecular velocity space may be still reduced up to one, by eliminating also $c_{x}$ velocity and so by reducing the computational efforts. However in this study we keep two velocities $\left(c_{x}, c_{y}\right)$ formulation, previously used for the steady state Couette and Fourier flows. It is shown below that we obtained the grid convergency in both physical and molecular velocity spaces with the reasonable number of the grid points and, therefore, computational time.

First, the discrete velocity method (DVM) is applied to split the continuum $(-\infty, \infty)$ molecular velocity space $c_{x}, c_{y}$ in the governing equation (16) into discrete velocity sets $c_{x_{m}}, c_{y_{k}}$ where $m, k=-N_{c}, . .,-1,1,2, . ., N_{c}$. These velocities $c_{x_{m}}, c_{y_{k}}$ are taken to be the roots of the Hermite polynomial of order $N_{c}$. Then the set of $2 N_{c}$ kinetic equations, corresponding to $2 N_{c}$ values of discrete velocities, is discretized in time and space by finite difference method (FDM). Here $N_{c}$ is taken to be equal to 20 . Grid-independence in molecular velocity space is checked with a finer grid of $50 \times 50$ points showing less than $1 \%$ difference in all macroscopic profiles.

The spacial derivatives are approximated by the second-order accurate TVD type scheme as in [28]. The number of uniformly distributed points in physical space $N_{y}$ is equal to 500 . The time derivative is approximated by the timeexplicit Euler method. The time step, $\Delta t$, is chosen according to the following condition:

$$
\Delta t=C F L / \max \left\{\gamma_{1} A, \gamma_{2} A, c_{y}^{N_{c}} / \Delta y\right\},
$$

where $C F L$ is the Courant-Friedrichs-Lewy number which is equal here to 0.5. With the chosen computational grid the last term $\left(c_{y}^{N_{c}} / \Delta y\right)$ in condition (22) is preponderant, therefore the simulations for both mixtures are carried out with the same time step $\Delta t=0.77 \cdot 10^{-4}$.

For the present simulations the termination condition is defined for the heat flux in following form

$$
\min _{i}\left|q_{i}^{(l+1)}-q_{i}^{(l)}\right| /\left|q_{i}^{(l)}\right| \leq \varepsilon
$$

where $l$ is the number of time step, $i=1, N_{y}$, and here $\varepsilon=10^{-7}$ is used. The termination condition, Eq. (23), is checked for every macroscopic parameter and it is found that the higher order moments like the heat flux, converge more rapidly as the lower one. However we focus our study on the heat flux evolution in time, so the termination condition is applied for the heat flux. The time needed to reach criterion (23) is designated as $t_{\varepsilon}$.

\section{Results and discussion}

Two gas mixture of the noble gases with similar $m^{A r} / m^{N e}=1.979$ and disparate $m^{A r} / m^{H e}=9.970$ molecular masses are considered. The calculations were carried out for three values of concentration: $C_{0}=0.1,0.5$ and 0.9 and for the rarefaction parameter equal to $\delta=0.1,1$ and 10 . The molecular diameter 
ratio is needed to calculate the collision term in Eq. (11). Here, in the frame of the Hard Sphere molecular model [29] the following data for the diameter ratio $d^{A r} / d^{N e}=1.406$ and $d^{A r} / d^{H e}=1.668$ were used.

\subsection{Transient behaviors of the macroscopic parameters}

Initially, at $t=0$ the gas mixture is in its equilibrium state at temperature $T_{0}$, then impulsively the temperature of the upper (down) plate increases (decreases) to value $T_{0}+\Delta T / 2\left(T_{0}-\Delta T / 2\right)$, respectively. The sudden heating of upper plate and cooling of down plate causes a decrease (increase) in the number density near the upper (down) plate, respectively. This local difference in the mixture number density creates a mass flow of a gas from the upper plate to the down plate and then backwards. Consequently, we observe the number density and macroscopic velocity disturbances propagate in a wave-like manner along the domain; after several time the waves are damped and the macroscopic velocity vanishes.

The transient behaviors of the mixture macroscopic quantities (number density, macroscopic velocity, temperature, and concentration) are represented on Figs. $2-5$ for two mixtures Ne-Ar and He-Ar, three concentrations of the light species $C_{0}=0.1,0.5$ and 0.9 and for various values of the rarefaction parameter ranging from the near free molecular to the slip flow regime. The macroscopic parameters are shown as a function of the dimensionless time, defined in Eq. (12). The behaviors of the mixture macroscopic parameters are symmetric (macroscopic velocity and heat flux) and anti-symmetric (number density, temperature and concentration) relatively to the half distance between the plates. Therefore only the data near the hot (upper) plate are plotted, except for the macroscopic velocity, which is taken at the middle point between the plates.

The variations in time of the mixture number density and mixture macroscopic velocity are shown on Figs. 2 (for Ne-Ar mixture) and 3 (for He-Ar mixture), for three values of rarefaction parameter and three concentrations. The number density and velocity behaviors depend strongly on the gas rarefaction. For the higher rarefaction level, $\delta=0.1$ and 1 , the new equilibrium state of the gas mixture is reached through the decaying waves propagating across the gap. The gas mixture moves from the hotter plate toward the colder one and then vise versa to achieve finally the new equilibrium state, where the macroscopic velocity vanishes. Different behaviors of the number density and macroscopic velocity are observed in slip flow regime, $\delta=10$, the wave structure movement does not appear anymore and only monotone decreasing in the number density is observed with the macroscopic gas movement from the hotter to the colder plate, see Figs. 2 and 3.

The composition of Ne-Ar mixture does not affect practically the number density and macroscopic velocity transient behaviors. In contrast, for the He-Ar mixture, the composition impacts essentially the intensity of the macroscopic velocity, which reaches a maximum for the lower value of concentration in the near free molecular and transitional flow regimes. 
Figure 4 gives information about the time evolution of He-Ar mixture temperature. As it was seen for the time evolution of the mixture number density and macroscopic velocity, see Figs. 2 and 3, the temperature time evolution depends strongly on the gas rarefaction. After a sudden heating of the upper plate the gas temperature near the wall increases up to its maximum value and then decreases with time up to its equilibrium value. This behavior is observed for $\delta=0.1$ and 1 . The temperature jump between the gas and surface temperatures is clearly seen and it increases with rarefaction parameter decreases. Contrarily, in the slip flow regime, $\delta=10$, the temperature increases monotonically with time up to its equilibrium value and the temperature jump is much smaller than that in more rarefied conditions. The mixture nature and mixture composition have only slight impact on the transient behavior of the temperature.

The transient behaviors of the light species concentration are shown on Fig. 5 for Ne-Ar and He-Ar mixtures, three initial concentration and various flow regimes. As for the mixture number density, the wave-like structure of the concentration time evolution can be observed with larger number of the waves compared to the number density. The concentration behavior depends strongly on the gas rarefaction. For the higher gas rarefaction, $\delta=0.1$, the new equilibrium value of the concentration near the hot plate is close to its initial state while with rarefaction decreasing essential rise in the lighter species concentration is observed near the hotter plate. As expected, the time behavior of the concentration depends strongly on the mixture composition and the nature of the mixture.

\subsection{Time dependent macroscopic profiles in the gap between the plates}

Typical time dependent profiles of the mixture number density, macroscopic velocity and concentration in the gap between the plates are shown in Figs. 6 8 for $C_{0}=0.5$ and $\delta=1$ and 10, for He-Ar mixtures, respectively. As it is clear from Figs. 6, the time evolution of the mixture number density profiles show the same trend as in Fig. 3: in the early time the number density decreases (increases) only near the hot (cold) plate, while the rest of the profile remains unchanged. Then, the number density profile converges, but non-monotonically, to the typical mixture number density profile, see Ref. [30]. The species number density evolutions in the gap are shown on Fig. 6 (down line) for $\delta=0.1$ : it is clear that the lighter species He $\left(n_{1}\right)$ takes less time, compared to heavier one, $\operatorname{Ar}\left(n_{2}\right)$, to converge to its steady state profile.

The wave-like structure can be observed in Figs. 7 and 8 for the timedependent mixture' macroscopic velocity profiles and the profiles of the concentration. The mixture velocity profile tends non-monotonically to zero, see Fig. 7 top line for $\delta=1$ and 10. Figure 7, down line, demonstrates the time evolution of the macroscopic velocity for each species for $C_{0}=0.5$ and $\delta=1$. The perturbation wave velocity of $\mathrm{He}$ at $t=0.03$ is more than 3 times larger than that of $A r$, which can be explained by the difference in the most probable speed of each species, while the macroscopic velocity of the lighter species $(\mathrm{He}$, $u_{1}$ ) relaxes faster than the heavier one $\left(A r, u_{2}\right)$. 
The concentration profiles, shown on Fig. 8 for $\delta=1$ and 10, converge also non-monotonically to the typical steady state concentration distribution, where the concentration of the lighter species increases (decreases) near the hot (cold) plate.

\subsection{Heat flux response time}

The response time of a media is very important characteristic and it can be useful in the metrology, for example for the development of the fast response sensors. The steady state values of the heat flux between the plates, obtained after the sudden surfaces temperature changes, are provided in Table 1 for two mixtures, three concentrations and three values of the rarefaction parameter. The comparison between the heat flux, provided in Table 1, and similar results from Ref. [10], where the steady-state numerical approach was applied, shows the maximal difference approximately $1 \%$, which is of the order of the reported in Ref. [10] accuracy. From Table 1 it can be seen that for the fixed gas rarefaction, whatever is the flow regime, the heat flux depends slightly on the initial mixture composition for the mixture of the gases with similar molecular masses (Ne-Ar), however for the gas mixture with disparate molecular masses (He-Ar) the mixture composition affects the heat flux.

\begin{tabular}{|c|c|c|c|c|c|c|}
\hline \multirow{3}{*}{$\delta$} & \multicolumn{6}{|c|}{$-q_{y}$} \\
\cline { 2 - 7 } & \multicolumn{3}{|c|}{$\mathrm{Ne}-\mathrm{Ar}$} & \multicolumn{3}{|c|}{$\mathrm{He}-\mathrm{Ar}$} \\
\cline { 2 - 7 } & $C_{0}=0.1$ & 0.5 & 0.9 & 0.1 & 0.5 & 0.9 \\
\hline 0.1 & 0.5430 & 0.5589 & 0.5446 & 0.6203 & 0.8261 & 0.6890 \\
\hline 1 & 0.4058 & 0.4172 & 0.4068 & 0.4588 & 0.6089 & 0.5180 \\
\hline 10 & 0.1364 & 0.1396 & 0.1365 & 0.1498 & 0.1941 & 0.1733 \\
\hline
\end{tabular}

Table 1: The steady state mixture heat flux $q_{y}$ for various values of the concentration $C_{0}$ and the rarefaction parameter $\delta$, for two mixtures: Ne-Ar and He-Ar.

The transient heat flux near the hot surface is plotted on Fig. 9 for two mixtures and for three rarefaction parameters $\delta=0.1,1$ and 10 using now the logarithmic scale for time, so the initial transitional time can be better seen. As it is clear from Fig. 9, after the sudden surface temperature changes, the heat flux near the hot surface changes instantaneously from 0 to some value and than keeps this value during certain time. This initial value of the heat flux can be easily found from the simple physical reasoning. During a short time after sudden change of the wall temperature the heat flux is determined only by the distribution function of the molecules reflected from the wall. This time is sufficiently short, so the molecules coming from the opposite wall with different temperature cannot reach yet this wall. We can call here this short time "early steady state time", $t_{e}$. This time $t_{e}$ depends on the rarefaction level through mean collision time (or time-of-flight of molecules), $t_{c}$, Eq. (14).

From the reasoning provided above the heat flux value, which is kept during the early steady state time, is equal to the half of the heat flux in the free molecular limit. This free molecular value can be easily found analytically, see 
[29]. In the case of diffuse gas-surface interaction it becomes

$$
q_{y \alpha}^{\prime}=\frac{n_{\alpha} m_{\alpha}}{2 \sqrt{\pi}}\left(\frac{2 k T_{0}}{m_{\alpha}}\right)^{3 / 2} \frac{\Delta T}{T_{0}} .
$$

Using the dimensionless quantities, Eqs. (2) and (5), the early steady state heat flux of the mixture becomes:

$$
q_{y}=\frac{1}{2 \sqrt{\pi}}\left(C_{0} \sqrt{\frac{m}{m_{1}}}+\left(1-C_{0}\right) \sqrt{\frac{m}{m_{2}}}\right) .
$$

The "early steady state time", $t_{e}$, of the mixture heat flux is provided in Table 2 for two mixtures. This time is defined as a time needed for the heat flux to deviate from the analytical value, Eq. (25), in 1\%. As can be seen from Fig. 9 and Table 2 this time of quasi constant heat flux decreases with $\delta$ increases, decreasing as approximately $\frac{1}{\delta}$, so with the same tendency as the mean collision time changes with rarefaction level, Eq.(15). This time changes only slightly with the concentration changes for the case of Ne-Ar mixture, with a minimum for the concentration $C_{0}=0.5$. The same tendency with a minimum of time $t_{e}$ for the concentration equal to 0.5 is kept for the He-Ar mixture, but only for higher values of the rarefaction parameter.

The "early steady state time", $t_{e}$, of species heat flux in the case of He-Ar mixture is provided in Table 3. For all considered values of concentration and the rarefaction level the time $t_{e}$ for the lighter species $(\mathrm{He})$ is alway lower than that of the larger one (Ar) with the ratio $t_{e}^{A r} / t_{e}^{H e}$ of the order of 2-4.

During the early steady time the particles from the cold wall have not reached yet the hot wall and the solution is determined only by the molecules in the vicinity of the hot wall. These results are in agreement with the analytical solution obtained in [12] for a single gas and with the numerical results provided in [20] in the case of the gas mixture between two coaxial cylinders. This particularity of the heat flux evolution in time needs to be take into account when one estimates the response time of the various sensors.

\begin{tabular}{|c|c|c|c|c|c|c|}
\hline \multirow{3}{*}{$\delta$} & \multicolumn{3}{|c|}{$t_{e}$} \\
\cline { 2 - 7 } & \multicolumn{3}{|c|}{$\mathrm{Ne}-\mathrm{Ar}$} & \multicolumn{3}{c|}{$\mathrm{He}-\mathrm{Ar}$} \\
\cline { 2 - 7 } & $C_{0}=0.1$ & 0.5 & 0.9 & 0.1 & 0.5 & 0.9 \\
\hline 0.1 & 0.325 & 0.309 & 0.340 & 0.135 & 0.159 & 0.263 \\
\hline 1 & 0.0812 & 0.0765 & 0.0812 & 0.0464 & 0.0386 & 0.0618 \\
\hline 10 & 0.00580 & 0.00541 & 0.00580 & 0.00386 & 0.00271 & 0.00464 \\
\hline
\end{tabular}

Table 2: The "early steady state time", $t_{e}$, of the mixture heat flux for Ne-Ar and He-Ar mixtures, for different initial concentration $C_{0}$ and rarefaction parameter $\delta$.

Analyzing the evolution of the heat flux after this early steady time, see Fig. 9 , we can conclude that the heat flux relaxation speed $\mathrm{d} q_{y} / \mathrm{d} t$ near the hot plate to the final equilibrium state depends slightly on the species concentration for $\mathrm{Ne}-\mathrm{Ar}$ mixture. However, for the mixture of two species with largely different 


\begin{tabular}{|c|c|c|c|c|c|c|}
\hline \multirow{3}{*}{$\delta$} & \multicolumn{9}{|c|}{$t_{e}$} \\
\cline { 2 - 7 } & \multicolumn{2}{|c|}{$C_{0}=0.1$} & \multicolumn{2}{|c|}{0.5} & \multicolumn{2}{c|}{0.9} \\
\cline { 2 - 7 } & $\mathrm{He}$ & $\mathrm{Ar}$ & $\mathrm{He}$ & $\mathrm{Ar}$ & $\mathrm{He}$ & $\mathrm{Ar}$ \\
\hline 0.1 & 0.121 & 0.375 & 0.155 & 0.478 & 0.262 & 0.796 \\
\hline 1 & 0.0208 & 0.0866 & 0.0301 & 0.0974 & 0.0603 & 0.1183 \\
\hline 10 & 0.00155 & 0.00580 & 0.00193 & 0.00696 & 0.00425 & 0.00850 \\
\hline
\end{tabular}

Table 3: The "early steady state time", $t_{e}$, for He-Ar mixture, for different initial concentration $C_{0}$ and rarefaction parameter $\delta$.

masses $(H e-A r)$, see Fig. 9 (right column), the relaxation speed of heat flux is affected by the species concentration. The heat flux behavior depends strongly on the rarefaction level: the heat flux decreases with time for higher level of gas rarefaction $(\delta=0.1$ and 1$)$ and increases to its new equilibrium value for $\delta=10$

Figure 10 shows the heat flux profiles for Ne-Ar mixture with $C_{0}=0.5$ as a function of the position in the gap between the plates and for various time moments for two values of the rarefaction parameter $\delta=1$ and 10 . It can be seen from Fig. 10 that the transient behavior of the total heat flux depends on the distance to the plates and on the rarefaction level. For $\delta=1$ the heat flux decreases with time in the whole gap. However, for $\delta=10$, this behavior changes in the vicinity of the plates: the heat flux decreases up to a value and then increases to its new equilibrium state.

The time needed to reach the convergence criterion $(23), t_{\varepsilon}$, was obtained for different values of initial concentration $C_{0}$ and rarefaction parameter. The values of $t_{\varepsilon}$ are presented in Table 4 . It is interesting to note that for a fixed initial concentration $C_{0}$ this time $t_{\varepsilon}$ has a minimum value in the transitional flow regime whatever the concentration or the mixture composition. For the fixed level of the gas rarefaction the minimum $t_{\varepsilon}$ is reached for $C_{0}=0.5$ whatever the mixture composition.

\begin{tabular}{|c|c|c|c|c|c|c|}
\hline \multirow{3}{*}{$\delta$} & \multicolumn{6}{|c|}{$t_{\varepsilon}$} \\
\cline { 2 - 7 } & \multicolumn{3}{|c|}{$\mathrm{Ne}-\mathrm{Ar}$} & \multicolumn{3}{|c|}{$\mathrm{He}-\mathrm{Ar}$} \\
\cline { 2 - 7 } & $C_{0}=0.1$ & 0.5 & 0.9 & 0.1 & 0.5 & 0.9 \\
\hline 0.1 & 13.37 & 10.66 & 12.90 & 9.66 & 9.27 & 10.12 \\
\hline 1 & 4.40 & 4.25 & 4.32 & 4.40 & 4.32 & 4.40 \\
\hline 10 & 9.98 & 9.60 & 9.60 & 9.71 & 8.82 & 7.46 \\
\hline
\end{tabular}

Table 4: Time $t_{\varepsilon}$ needed to satisfy criterion (23) for different initial concentration $C_{0}$ and rarefaction parameter $\delta$.

More interesting from the practical point of view is to define a steady state time $t_{s}$ as a time needed for the heat flux to reach a value, which is different from the steady state value in $1 \%$. Table 5 gives the steady state time as a time needed for the heat flux in the gap to achieve a value, which is different in $1 \%$ from its steady state value, presented in Table 1 . For near continuum regime $(\delta=10)$ 
this steady state time decreases with increase of light component fraction. For higher level of gas rarefaction $(\delta=0.1$ and 1$)$ the time to reach the steady state has a maximum for $C_{0}=0.5$. For the Ne-Ar mixture this steady state time for $C_{0}=0.5$ is $3.8-4.4 \%$ higher compared to the concentration $C_{0}=0.1$. This difference in the steady state time increases for He-Ar mixture up to $20 \%$ for $\delta=0.1$. At the fixed value of concentration parameter $C_{0}$ steady state time $t_{s}$

\begin{tabular}{|c|c|c|c|c|c|c|}
\hline \multirow{3}{*}{$\delta$} & \multicolumn{5}{|c|}{$t_{s}$} \\
\cline { 2 - 7 } & \multicolumn{3}{|c|}{ Ne-Ar } & \multicolumn{3}{c|}{ He-Ar } \\
\cline { 2 - 7 } & $C_{0}=0.1$ & 0.5 & 0.9 & 0.1 & 0.5 & 0.9 \\
\hline 0.1 & 1.78 & 1.85 & 1.70 & 1.78 & 2.24 & 1.31 \\
\hline 1 & 1.70 & 1.78 & 1.70 & 1.78 & 2.08 & 1.23 \\
\hline 10 & 7.47 & 7.06 & 6.57 & 7.00 & 5.14 & 3.81 \\
\hline
\end{tabular}

Table 5: The steady state time, $t_{s}$, a time needed to obtain the value of the heat flux in the gap, which is different in $1 \%$ from its steady state value for different values of initial concentration $C_{0}$ and rarefaction parameter $\delta$.

takes the minimum value in transitional flow regime $(\delta=1)$. This behavior is similar to the results of Ref. [20], where the minimum of the steady state time was found in the transitional flow regime when the heat transfer through the gas mixture between two coaxial cylinders is analyzed. In the slip flow regime the steady state time decreases with increasing of the concentration of the lighter species and it is lower for the He-Ar mixture.

For the practical application it is interesting to have an idea on the order of magnitude of the steady state time at a given pressure. From the data provided in Table 5 we can estimate this time. Let us consider a small distance between the plates of $1 \mathrm{~mm}$ and the reference temperature $T_{0}$ equal to $300 \mathrm{~K}$. The mixture viscosity can be evaluated, for example, using the Wilke formula [31]. Then, from the given rarefaction parameter a pressure can be calculated and the corresponding steady state time. These values for He-Ar mixture and three concentrations are provided in Table 6 . For a fixed equilibrium concentration of

\begin{tabular}{|c|c|c|c|c|c|}
\hline \multicolumn{2}{|c|}{$C_{0}=0.1$} & \multicolumn{2}{c|}{0.5} & \multicolumn{2}{c|}{0.9} \\
\hline$p^{\prime}(\mathrm{Pa})$ & $t_{s}^{\prime},(\mu s)$ & $p^{\prime}(\mathrm{Pa})$ & $t_{s}^{\prime},(\mu s)$ & $p^{\prime}(\mathrm{Pa})$ & $t_{s}^{\prime},(\mu s)$ \\
\hline 0.854 & 4.80 & 1.13 & 4.70 & 1.81 & 1.62 \\
8.54 & 4.80 & 11.3 & 4.36 & 18.1 & 1.52 \\
85.4 & 18.89 & 113. & 10.79 & 181. & 4.70 \\
\hline
\end{tabular}

Table 6: The dimensional steady state time, $t_{s}^{\prime}$, for He-Ar mixture.

the lighter species $C_{0}$ significantly more time is needed for the heat flux establishment when pressure jumps over a threshold of around $100 \mathrm{~Pa}$. However for the similar values of pressure it is clear from Table 6 that increasing equilibrium concentration $C_{0}$ requires shorter time to establish the steady state. 


\section{Conclusion}

The transient heat transfer due to the sudden change of the walls' temperatures through a binary gas mixture was simulated on the basis of numerical solution of kinetic model equation with McCormack collision integral in a wide range of rarefaction parameter and species concentrations. The cases of small and large species mass ratio were considered. The time needed for the heat flux to reach its new equilibrium steady state condition, steady state time, was established for both mixtures, three concentrations and different rarefaction level. It was found that in the near free molecular and transitional flow regimes this steady state time has a maximum for the concentration equal to 0.5 , whatever is the mixture composition, while in the slip flow regime the steady state time decreases with increasing of the lighter species concentration. For the fixed value of the concentration the time has a minimum in the transitional flow regime. The particular behavior is observed just after the sudden temperature change: the heat flux keeps a constant value during 0.03 - 0.3 characteristic times depending on the gas rarefaction and then starts to evolve to new equilibrium state. The time evolution of macroscopic quantities, number density, macroscopic velocity, temperature and concentration, were obtained for both mixtures and in large rarefaction parameter range.

\section{Acknowledgement}

M. T. Ho and I. Graur thank the Labex MEC project (ANR-10-LABX-0092) and the A*MIDEX project (ANR-11-IDEX-0001-02), funded by the "Investissements d'Avenir" French Government program managed by the French National

Research Agency (ANR), for their support. A. Polikarpov thanks the Russian Science Foundation (project No. 1419-01755) for its financial support.

[1] D R Willis. Heat transfer in a rarefied gas between parallel plates at large temperature ratios. In J A Lauermann, editor, Rarefied Gas Dynamics, volume 1, pages 209-225. Academic Press, New York, 1963.

[2] P Bassanini, C Cercignani, and C D Pagani. Comparison of kinetic theory analysis of linearized heat transfer between parallel plates. Int. J. Heat Mass Transfer, 10:447-460, 1967.

[3] P Bassanini, C Cercignani, and C D Pagani. Influence of the accommodation coefficient on the heat transfer in a rarefied gas. Int. J. Heat Mass Transfer, V.11, pp. 1359-1368, 1968.

[4] J R Thomas, T S Chang, and Siewert C E. Heat transfer between parallel plates with arbitrary surface accommodation. Physics of Fluids, 16(12), 1973.

[5] T Ohwada, Y Sone, and K Aoki. Numerical analysis of the Poiseuille and thermal transpiration flows between two parallel plates on the basis of 
the Boltzmann equation for hard sphere molecules. Physics of Fluids A, 1(12):2042-2049, 1989.

[6] C E Siewert. Viscous-slip, thermal-slip and temperature-jump coefficients as defined by the linearized Boltzmann equation and the Cercignani-Lampis boundary condition. Phys. Fluids, 15(6):1696-1701, 2003.

[7] I A Graur and A Polikarpov. Comparison of different kinetic models for the heat transfer problem. Heat and Mass Transfer, 46:237-244, 2009.

[8] S Kosuge, K Aoki, and S Takata. Heat transfer in a gas mixture between two parallel plates: finite-difference analysis of the Boltzmann equation. In T J Bartel and M A Gallis, editors, Rarefied Gas Dynamics, volume 585, pages 289-296, Melvile, 2001. 22nd Int. Symp., AIP Conference Proc.

[9] R D M Garcia and C E Siewert. The McCormack model for gas mixtures: Heat transfer in a plane channel. Phys. Fluids, 16(9):3393-3402, 2004.

[10] F Sharipov, L M G Cumin, and D Kalempa. Heat flux through a binary gaseous mixture over the whole range of the knudsen number. Physica A, 378:183-193, 2007.

[11] Sone Y. Effect of sudden change of wall temperature in a rarefied gas. J. Phys. Soc. Jpn., 20(2):222-229, 1965.

[12] M Perlmutter. Analysis of transient heat transfer through a collisionless gas enclosed between parallel plates. ASME Paper 67-HT-53, 1967.

[13] D C Wadsworth, D A Erwin, and E Ph Muntz. Transient motion of a confined rarefied gas due to wall heating or cooling. Journal of Fluid Mechanics, 248(1):219, April 1993.

[14] A Manela and N. G. Hadjiconstantinou. On the motion induced in a gas confined in a small-scale gap due to instantaneous boundary heating. J. Fluid Mechanics, 593(11):453-462, 2007.

[15] A Manela and N. G. Hadjiconstantinou. Gas motion induced by unsteady boundary heating in a small-scale slab. Phys. Fluids, 20(11):117104(11 pages), 2008.

[16] T. Doi. The mccormack model for gas mixtures: Heat transfer in a plane channel. Journal of Heat Transfer, 133(9):022404(9 pages), 2011.

[17] O Buchina, M Vargas, S Stefanov, and D Valougeorgis. Transient micro heat transfer in a gas confined between parallel plates due to a sudden increase of the wall temperature. In 3rd Micro and Nano Flows Conference (MNF2011), Thesaloniki, Greece, August 22-24, 2011.

[18] I Graur, M T Ho, and M Wuest. Simulation of the transient heat transfer between two coaxial cylinders. Journal of Vacuum Science \& Technology A: Vacuum, Surfaces, and Films, 31(6):061603.1-9, 2013. 
[19] D Kalempa and F Sharipov. Numerical modelling of thermoacoustic waves in a rarefied gas confined between coaxial cylinders. Vacuum, 109:326-332, 2014.

[20] M Vargas, S Stefanov, and V Roussinov. Transient heat transfer flow through a binary gaseous mixture confined between coaxial cylinders. International Journal of Heat and Mass Transfer, 59:302-315, 2013.

[21] F J McCormack. Construction of linearized kinetic models for gaseous mixture and molecular gases. Phys. Fluids, 16:2095-2105, 1973.

[22] J H Ferziger and H G Kaper. Mathematical Theory of Transport Processes in Gases. North-Holland Publishing Company, Amsterdam, 1972.

[23] F Sharipov, L M G Cumin, and D Kalempa. Plane Couette flow of binary gaseous mixture in the whole range of the Knudsen number. Eur. J. Mech. B/Fluids, 23:899-906, 2004.

[24] S Naris, D Valougeorgis, F Sharipov, and D Kalempa. Discrete velocity modelling of gaseous mixture flows in mems. Superlattices and Microstructures, 35(3-6):629 - 643, 2004.

[25] C K Chu. Kinetic-theoretic description of the formation of a shock wave. Phys. of Fluids, 12(8):12-22, 1965.

[26] A B Huang and Hartley D L. Kinetic theory of the sharp leading edge problem in supersonic flow. Phys. of Fluids, 96(12):96-108, 1969.

[27] P Andries and B Perthame. The es-bgk model equation with correct prandtl number. In Rarefied Gas Dynamics, AIP Conference Proceedings, volume 585, pages 30-36, 2001.

[28] A.Ph. Polikarpov and I. Graur. Unsteady rarefied gas flow through a slit. Vacuum, 101:79 - 85, 2014.

[29] G A Bird. Molecular Gas Dynamics and the Direct Simulation of Gas Flows. Oxford Science Publications, Oxford University Press Inc., New York, 1994.

[30] M T Ho, L Wu, I Graur, Y Zhang, and Reese J M. Comparative study of the boltzmann and mccormack equations for couette and fourier flows of binary gaseous mixtures. Int. J. Heat Mass Transfer, 96:29-41, 2016.

[31] C R Wilke. A viscosity equation for gas mixture. Journal Chem. Phys., 18(4):517-522, 1950.

[32] E M Shakhov. Generalization of the Krook kinetic relaxation equation. Fluid Dyn., 3(5):95-96, 1968.

[33] F Sharipov and D Kalempa. Gaseous mixture flow through a long tube at arbitrary Knudsen number. J. Vac. Sci. Technol. A, 20(3):814-822, 2002. 

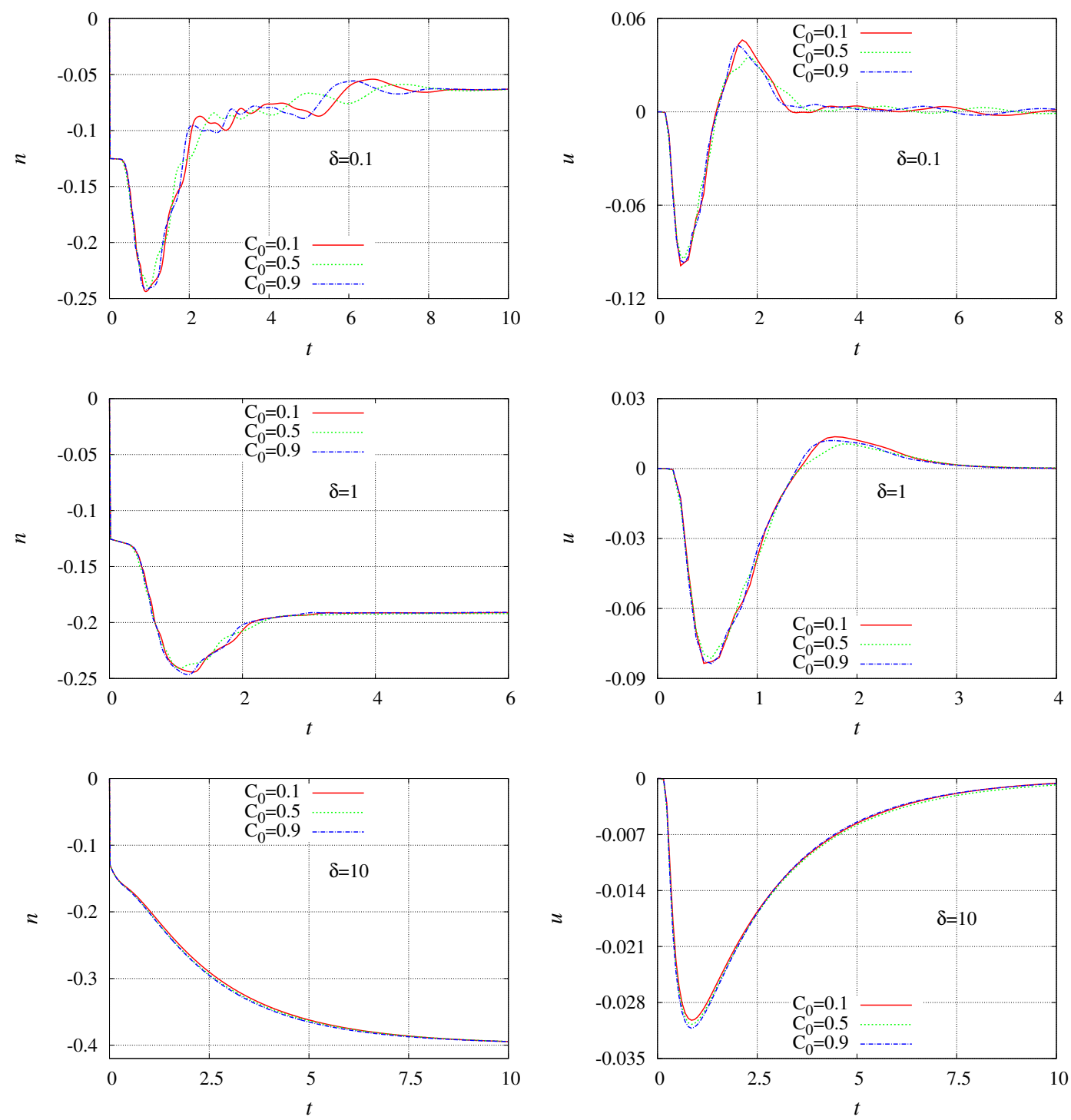

Figure 2: Time evolution of number density near the hot plate (left column) and the macroscopic velocity in the middle of the gap (right column) for mixture $\mathrm{Ne}-\mathrm{Ar}$, three concentrations and three values of the rarefaction parameter. 

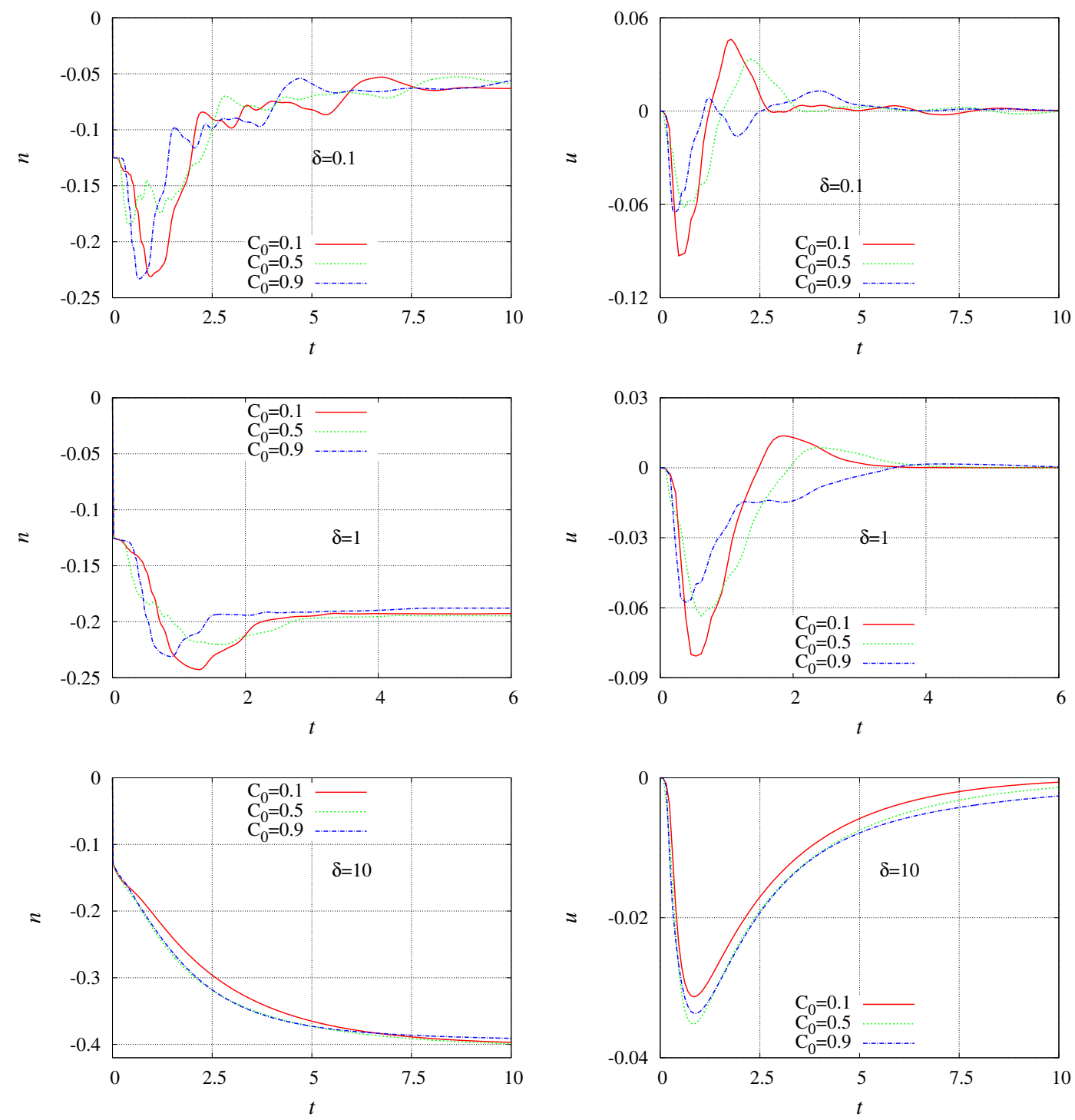

Figure 3: Time evolution of number density near the hot plate (left column) and the macroscopic velocity in the middle of the gap (right column) for mixture $\mathrm{He}-\mathrm{Ar}$, three concentrations and three values of the rarefaction parameter. 

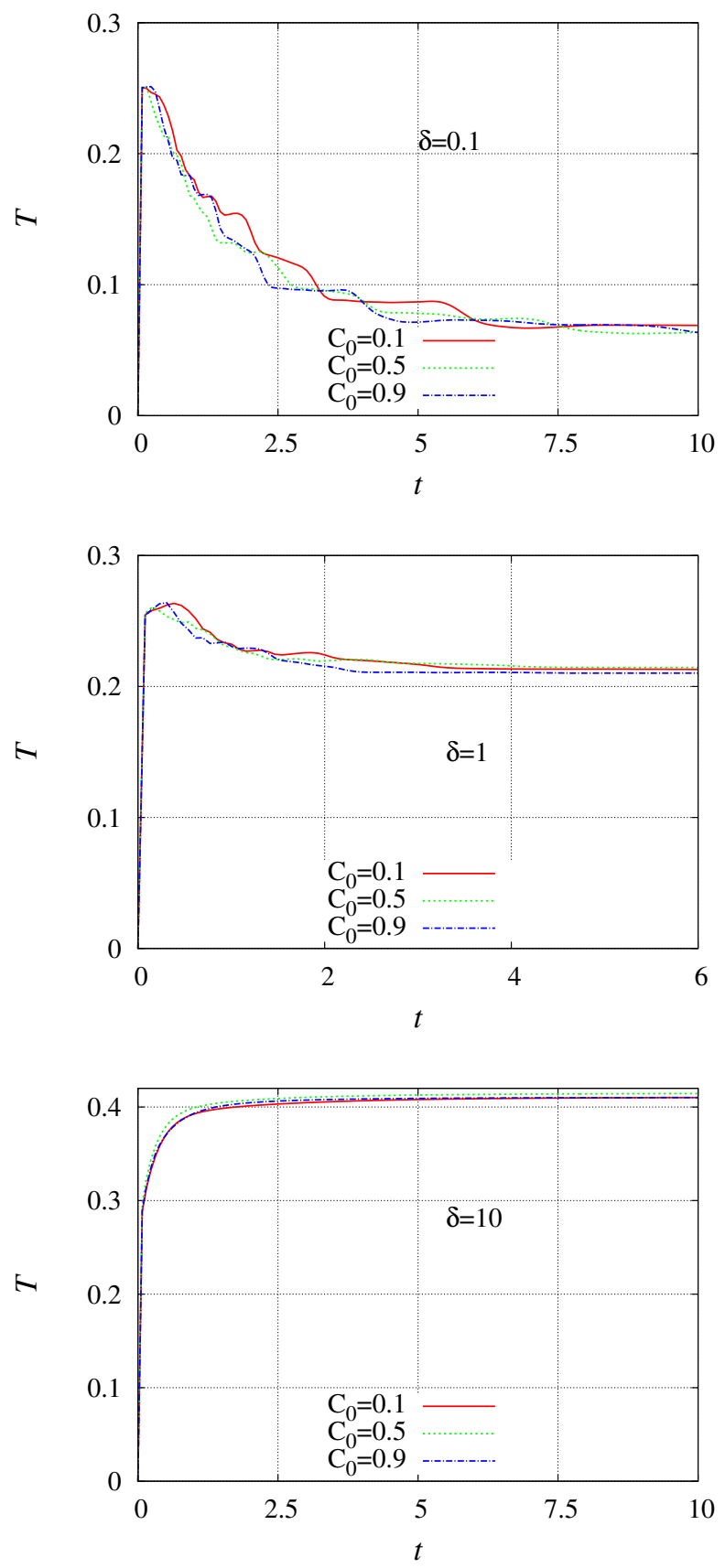

Figure 4: Time evolution of gas temperature near the hot plate for $\mathrm{He}-\mathrm{Ar}$ mixture, three values of the concentration and three values of the gas rarefaction parameter. 

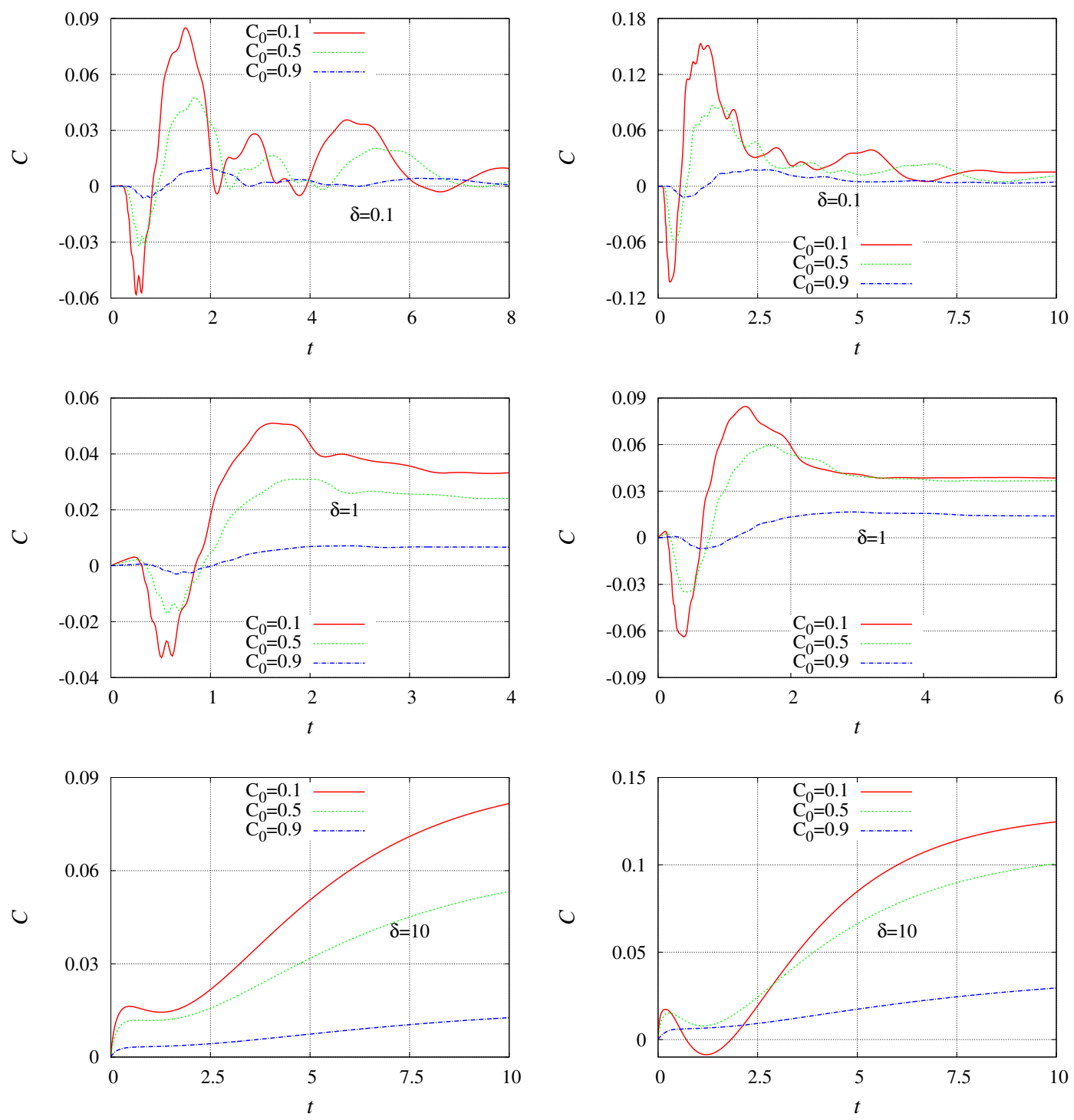

Figure 5: Time evolution of the concentration near the hot plate for the $N e-A r$ mixture (left column) and $\mathrm{He}-\mathrm{Ar}$ mixture (right column), for three values of the initial concentration and three rarefaction parameters. 

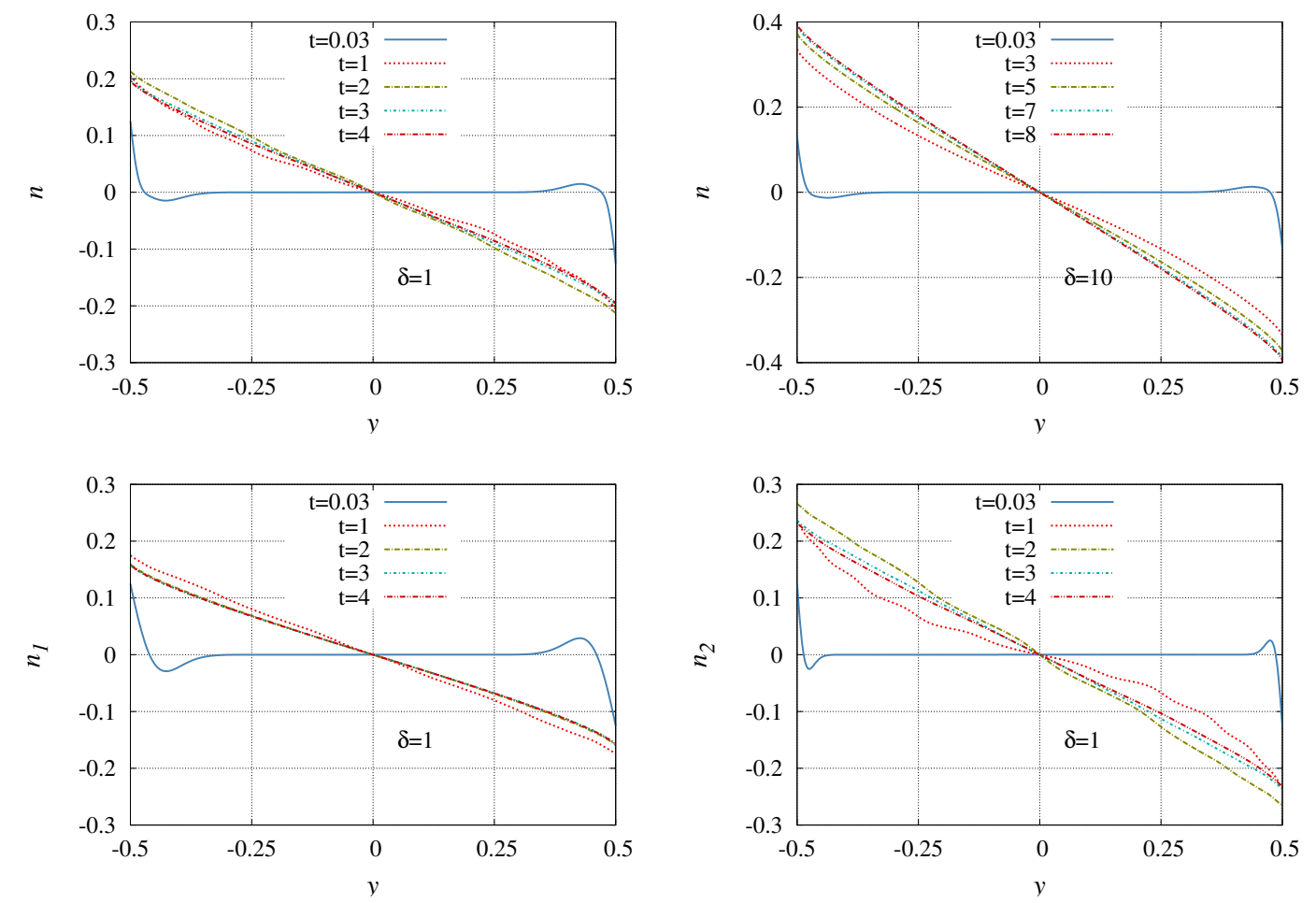

Figure 6: Top line: profiles of mixture number density between the plates for $\mathrm{He}-\mathrm{Ar}$ mixture, $C_{0}=0.5 ; \delta=1$ and 10; down line: the species number densities evolution, $\operatorname{He}\left(n_{1}\right)$ and $\mathrm{Ar}$ $\left(n_{2}\right)$ for $\delta=1$ and $C_{0}=0.5$. 

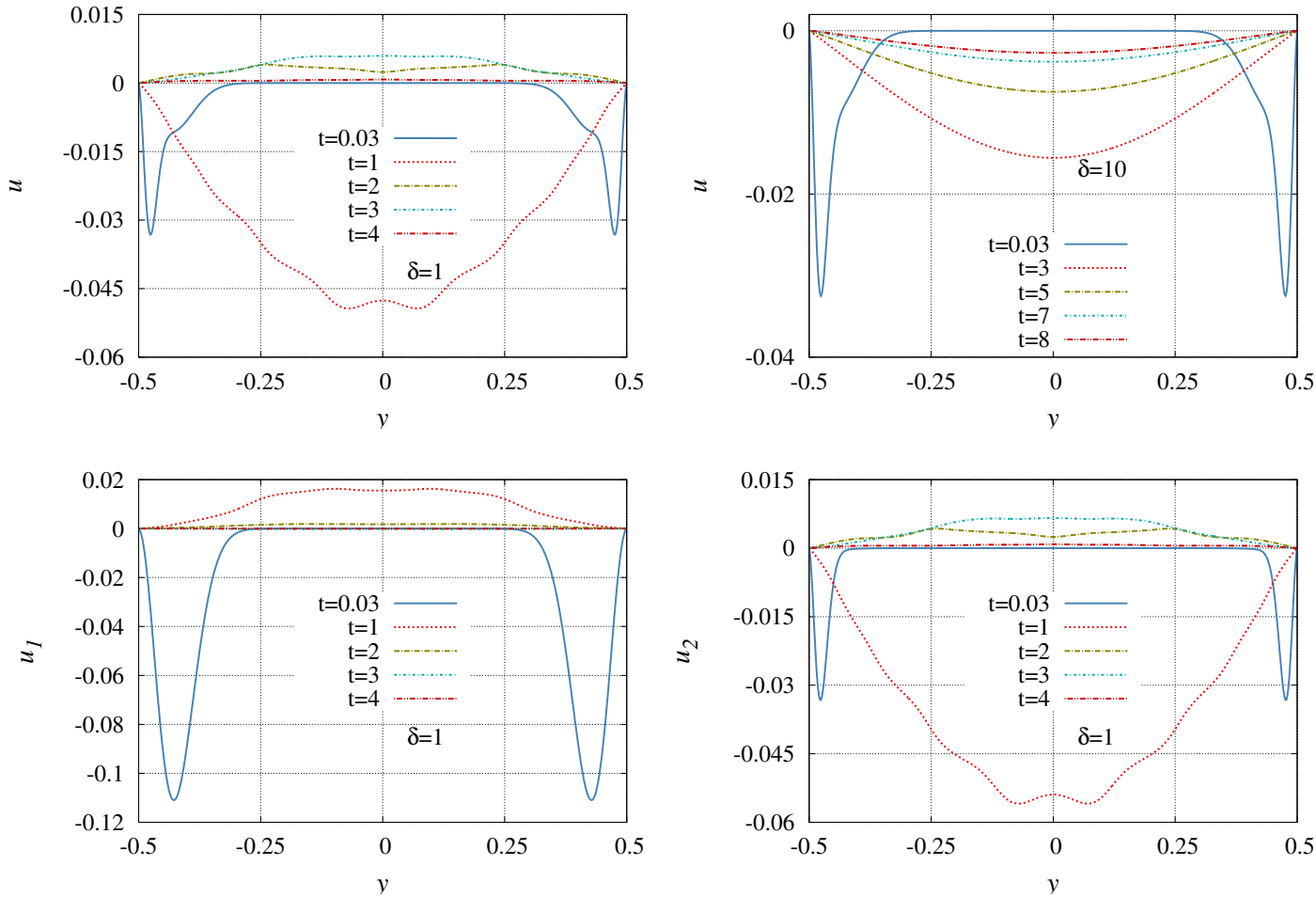

Figure 7: Top line: profiles of mixture macroscopic velocity between the plates for $\mathrm{He}-\mathrm{Ar}$ mixture, $C_{0}=0.5 ; \delta=1$ and 10; down line: the species macroscopic velocity evolution, $\mathrm{He}$ $\left(u_{1}\right)$ and $\operatorname{Ar}\left(u_{2}\right)$ for $\delta=1$ and $C_{0}=0.5$.
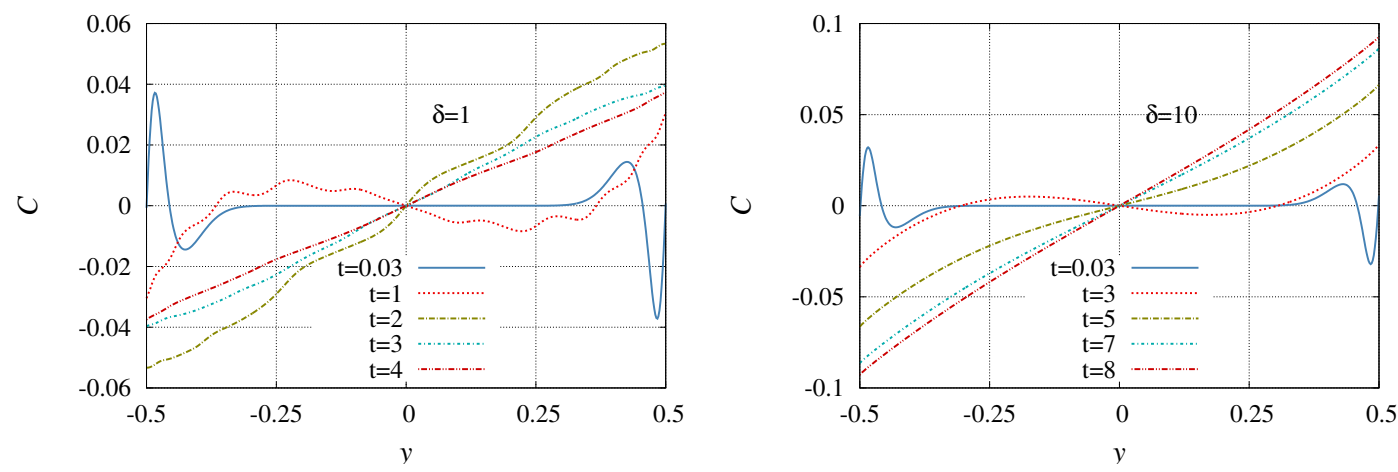

Figure 8: Profiles of concentration between the plates for $\mathrm{He}-\mathrm{Ar}$ mixture and $\mathrm{C}_{0}=0.5$ 

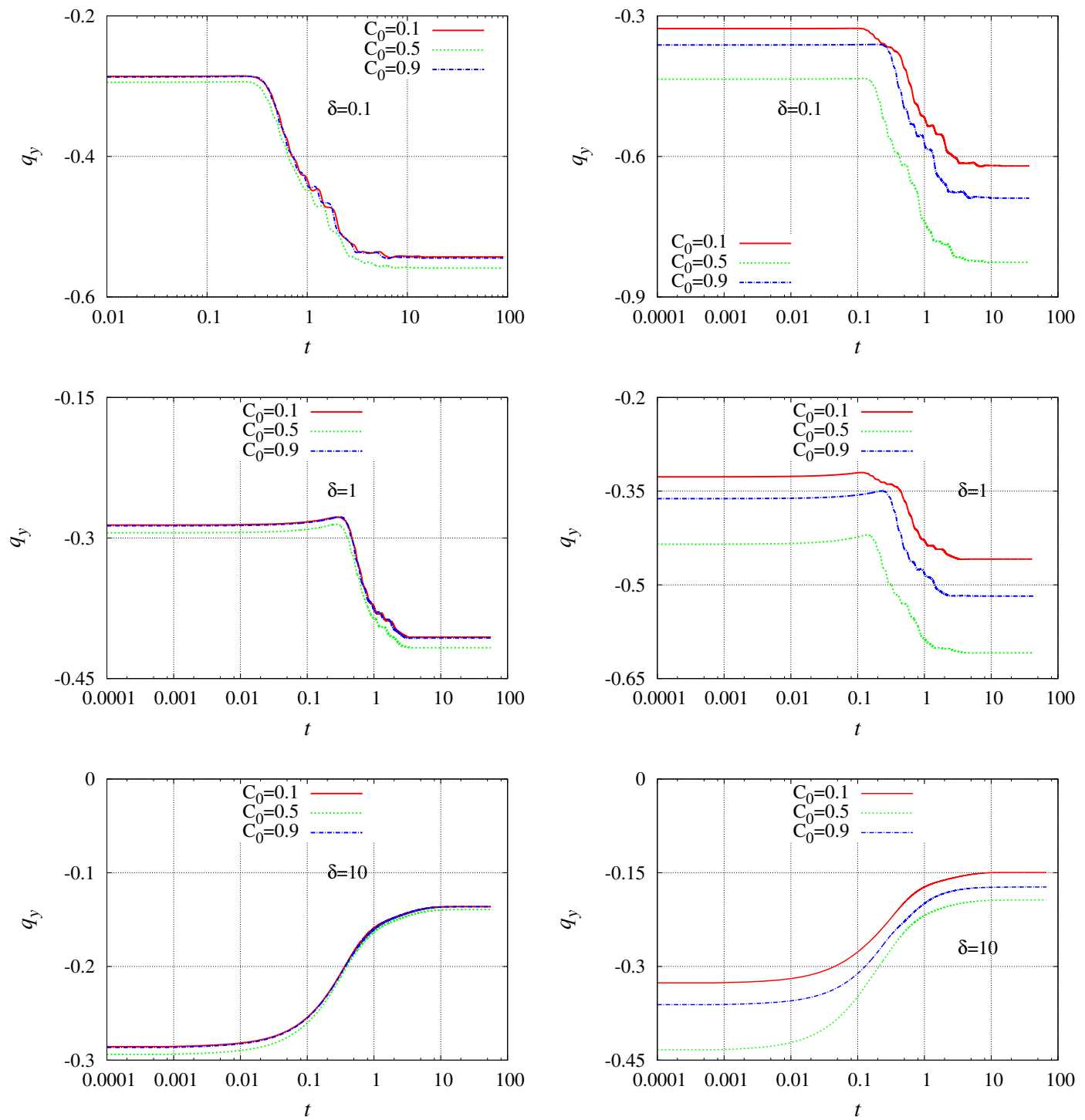

Figure 9: Time evolution of normal heat flux near the hot plate for two gas mixtures $\mathrm{Ne}-\mathrm{Ar}$ (left) and $\mathrm{He}-\mathrm{Ar}$ (right) using logarithmic scale in time. 

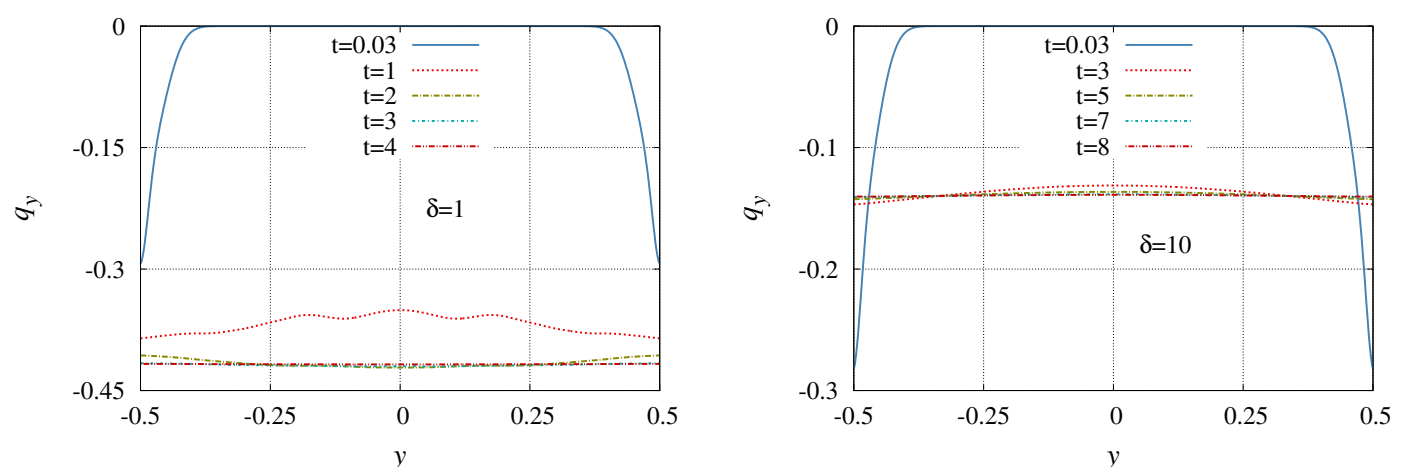

Figure 10: Profiles of normal heat flux between the plates for $N e-A r$ mixture and $C_{0}=0.5$

\section{Appendix A. The McCormack collision term}

The dimensionless collision term of the McCormack model [21] in eq. (16) has the following form:

$$
\begin{aligned}
\hat{L}_{\alpha \beta} h= & -\gamma_{\alpha \beta} h_{\alpha}+\gamma_{\alpha \beta} n_{\alpha} \\
& +2 \sqrt{\frac{m_{\alpha}}{m}}\left[\gamma_{\alpha \beta} u_{\alpha y}-v_{\alpha \beta}^{(1)}\left(u_{\alpha y}-u_{\beta y}\right)-\frac{v_{\alpha \beta}^{(2)}}{2}\left(q_{\alpha y}-\frac{m_{\alpha}}{m_{\beta}} q_{\beta y}\right)\right] c_{\alpha y} \\
& +\left[\gamma_{\alpha \beta} T_{\alpha}-2 \frac{m_{\alpha \beta}}{m_{\beta}}\left(T_{\alpha}-T_{\beta}\right) v_{\alpha \beta}^{(1)}\right]\left(c_{\alpha}^{2}-\frac{3}{2}\right) \\
& +2\left[\left(\gamma_{\alpha \beta}-v_{\alpha \beta}^{(3)}\right) P_{\alpha y y}+v_{\alpha \beta}^{(4)} P_{\beta y y}\right]\left(c_{\alpha y}^{2}-\frac{1}{2} c_{\alpha x}^{2}-\frac{1}{2} c_{\alpha z}^{2}\right) \\
& +\frac{4}{5} \sqrt{\frac{m_{\alpha}}{m}}\left[\left(\gamma_{\alpha \beta}-v_{\alpha \beta}^{(5)}\right) q_{\alpha y}+v_{\alpha \beta}^{(6)} \sqrt{\frac{m_{\beta}}{m_{\alpha}}} q_{\beta y}-\frac{5}{4} v_{\alpha \beta}^{(2)}\left(u_{\alpha y}-u_{\beta y}\right)\right] c_{\alpha y}\left(c_{\alpha}^{2}-\frac{5}{2}\right),
\end{aligned}
$$


where $\alpha, \beta=1,2$, and $v_{\alpha \beta}^{(i)}$ are defined as following

$$
\begin{aligned}
v_{\alpha \beta}^{(1)} & =\frac{16}{3} \frac{m_{\alpha \beta}}{m_{\alpha}} n_{\beta} \Omega_{\alpha \beta}^{11}, \\
v_{\alpha \beta}^{(2)} & =\frac{64}{15}\left(\frac{m_{\alpha \beta}}{m_{\alpha}}\right)^{2} n_{\beta}\left[\Omega_{\alpha \beta}^{12}-\frac{5}{2} \Omega_{\alpha \beta}^{22}\right], \\
v_{\alpha \beta}^{(3)} & =\frac{16}{5} \frac{m_{\alpha \beta}^{2}}{m_{\alpha} m_{\beta}} n_{\beta}\left[\frac{10}{3} \Omega_{\alpha \beta}^{11}+\frac{m_{\beta}}{m_{\alpha}} \Omega_{\alpha \beta}^{22}\right], \\
v_{\alpha \beta}^{(4)} & =\frac{16}{5} \frac{m_{\alpha \beta}^{2}}{m_{\alpha} m_{\beta}} n_{\beta}\left[\frac{10}{3} \Omega_{\alpha \beta}^{11}-\Omega_{\alpha \beta}^{22}\right], \\
v_{\alpha \beta}^{(5)} & =\frac{64}{15}\left(\frac{m_{\alpha \beta}}{m_{\alpha}}\right)^{3} \frac{m_{\alpha}}{m_{\beta}} n_{\beta}\left[\Omega_{\alpha \beta}^{22}+\left(\frac{15}{4} \frac{m_{\alpha}}{m_{\beta}}+\frac{25}{8} \frac{m_{\beta}}{m_{\alpha}}\right) \Omega_{\alpha \beta}^{11}-\frac{1}{2} \frac{m_{\beta}}{m_{\alpha}}\left(5 \Omega_{\alpha \beta}^{12}-\Omega_{\alpha \beta}^{13}\right)\right], \\
v_{\alpha \beta}^{(6)} & =\frac{64}{15}\left(\frac{m_{\alpha \beta}}{m_{\alpha}}\right)^{3}\left(\frac{m_{\alpha}}{m_{\beta}}\right)^{3 / 2} n_{\beta}\left[-\Omega_{\alpha \beta}^{22}+\frac{55}{8} \Omega_{\alpha \beta}^{11}-\frac{5}{2} \Omega_{\alpha \beta}^{12}+\frac{1}{2} \Omega_{\alpha \beta}^{13}\right],
\end{aligned}
$$

where

$$
m_{\alpha \beta}=\frac{m_{\alpha} m_{\beta}}{m_{\alpha}+m_{\beta}}
$$

is the reduced mass of the binary mixture. Note that $\Omega_{\alpha \beta}^{(i, j)}$ in Eq. (A.2) represents the omega integral [22], which for the case of the HS model is defined as $[22]$

$$
\Omega_{\alpha \beta}^{(i, j)}=\frac{(j+1) !}{8}\left[1-\frac{1+(-1)^{i}}{2(i+1)}\right]\left(\frac{\pi k T}{2 m_{\alpha \beta}}\right)^{1 / 2}\left(d_{\alpha}+d_{\beta}\right)^{2} .
$$

Finally, the parameters $\gamma_{\alpha \beta}$ are proportional to the collision frequency between species $\alpha$ and $\beta$ and appear in the collision term (A.1) only in the combinations $\gamma_{1}=\gamma_{11}+\gamma_{12}$ and $\gamma_{2}=\gamma_{21}+\gamma_{22}$, so one has only to define $\gamma_{1}$ and $\gamma_{2}$. The collision frequencies and the viscosity can be related in the same manner as in the Shakhov kinetic equation $[32,33,23]$ :

$$
\gamma_{\alpha}=\frac{p_{0_{\alpha}}}{\mu_{\alpha}}
$$

where $p_{0_{\alpha}}=n_{0_{\alpha}} k T_{0}$ is the equilibrium partial pressure and $\mu_{\alpha}$ is the partial viscosity given as

$$
\mu_{\alpha}=p_{0_{\alpha}} \frac{S_{\beta}+v_{\alpha \beta}^{(4)}}{S_{\alpha} S_{\beta}-v_{\alpha \beta}^{(4)} v_{\beta \alpha}^{(4)}}, \quad S_{\alpha}=v_{\alpha \alpha}^{(3)}-v_{\alpha \alpha}^{(4)}+v_{\alpha \beta}^{(3)}, \quad \text { and } \quad \beta \neq \alpha .
$$

Other details on the dimensionless presentation of omega integrals (A.4) and $v_{\alpha \beta}^{(i)}$ functions (A.2), as well as $\gamma_{\alpha \beta}$ and $S_{\alpha}$ can be found in [30]. 